\title{
Revisión de la sección Brevispicae del género Sloanea (Elaeocarpaceae) en Bolivia
}

\section{Revision of the section Brevispicae genus Sloanea (Elaeocarpaceae) in Bolivia}

\author{
Leonardo Palacios-Duque \& Carlos M. Baeza
}

Departamento de Botánica, Facultad de Ciencias Naturales y Oceanográficas, Universidad de Concepción, Casilla 160-C, Concepción, Chile.

lepduque@hotmail.com.

\begin{abstract}
RESUMEN
Se presenta un catálogo comentado e ilustrado de las especies de la sección Brevispicae del género Sloanea para Bolivia, se reconocen siete especies (S. eichlerii, S. guianensis, S. obtusifolia, S. picapica, S. pubescens, S. spathulata y S. tuerckheimii). Se presenta una clave para separar las especies y para cada una se proporcionan comentarios sobre afinidades taxonómicas, material tipo, distribución, hábitat, nombres comunes y usos.
\end{abstract}

Palabras Clave: Corymbo-racemi, Elaeocarpaceae, Flora de Bolivia, Paniculi, Sloanea, taxonomía.

\begin{abstract}
A critical checklist and illustrated of the species of section Brevispicae of the genus Sloanea is presented for Bolivia, seven species are recognized (S. eichlerii, S. guianensis, S. obtusifolia, S. picapica, S. pubescens, S. spathulata and $S$. tuerckheimii). A key to separate species is presented and for each are provide comments on taxonomic affinities, material type, distribution, habitat, common names and uses.
\end{abstract}

KeYwords: Corymbo-racemi, Elaeocarpaceae, Flora of Bolivia, Paniculi, Sloanea, taxonomy.

\section{INTRODUCCIÓN}

El género Sloanea L. está constituido por aproximadamente 150 especies distribuidas en los trópicos y subtrópicos del globo, aunque no está presente en África continental (Smith 2001). Las especies neotropicales, se encuentran desde México hasta el sur de Brasil y algunas islas del Caribe, se caracterizan por ser generalmente grandes árboles u ocasionalmente pequeños arbustos, con o sin raíces tabulares. Las hojas son alternas u opuestas, con pecíolos bipulvinulados; las estípulas pueden ser caedizas o persistentes. Las inflorescencias son principalmente racemosas, menos frecuentemente cimosas; las flores son apétalas en la mayoría de las especies. Los frutos son cápsulas leñosas, dehiscentes, generalmente cubiertas con espinas o en algunos casos inermes (Smith 1954, Alfaro 1981, Steyermark 1988, Smith \& Steyermark 1998, Smith 2001). De acuerdo con el número de sépalos presentes en la flor, Smith (1954) divide las especies neotropicales en dos subgéneros: las especies con cuatro sépalos iguales en forma y tamaño y que además cubren los órganos reproductivos antes de la antesis, forman el subgénero Quadrisepala Earle $\mathrm{Sm}$. (secciones Corymbo-racemi Earle Sm. y Paniculi Earle $\mathrm{Sm}$.) y las que poseen 4 a 11 sépalos desiguales en forma y tamaño y que no cubren completamente los órganos reproductivos antes de la antesis, constituyen el subgénero Sloanea Earle Sm. (secciones Brevispicae Earle Sm. y Sloanea Earle Sm.). En el anterior subgénero, las especies de la sección Sloanea presentan estípulas grandes $(1,5-15 \mathrm{~cm}$ de longitud), con venación de primer y segundo orden muy visible, además estas estípulas persisten con el desarrollo de las hojas, las inflorescencias son de gran tamaño, con las flores bien separadas y los frutos son generalmente grandes (más de 3,5 $\mathrm{cm}$ de longitud), provistos de espinas grandes y ligeramente recurvadas o completamente enroscadas (Smith 1954, Smith 1996, Palacios-Duque 2009, Palacios-Duque et al. 2012). La sección Brevispicae cuenta con cerca de 38 especies, caracterizadas por presentar estípulas pequeñas, rápidamente caedizas con el desarrollo de las hojas; las inflorescencias son pequeñas y compactas; los frutos son generalmente pequeños, con espinas cortas y rectas (Smith 1954, Palacios-Duque 2009, Palacios-Duque \& Fernández 
Alonso 2012). De acuerdo con revisiones totales o parciales del género Sloanea en el neotrópico, los países con mayor número de especies de la sección son: Perú (18), Colombia (15), Brasil (14), Venezuela (14), Costa Rica (9), Ecuador (8), Nicaragua (4) y Panamá con tres especies (Smith 1965, Alfaro 1981, Steyermark 1988, Liesner 1993, Smith \& Steyermark 1998, Jaramillo 1999, Smith 2001, Zamora et al. 2004, Palacios-Duque 2009, Vásquez 2009, Sampaio 2009, 2010). Sin embargo, para Bolivia aún se reconocen las tres especies citadas por Smith en 1954 (Sloanea gracilis Uitt., Sloanea guianensis (Aubl.) Benth., y Sloanea pubescens (Poepp. \& Endl.) K Schum.). Además, en los principales herbarios de Bolivia existen nuevas colecciones de Sloanea y muchas de ellas se encuentran sin determinar o mal determinadas. Por lo cual se dio inicio al estudio crítico de estas colecciones y es así como en este trabajo se presenta un catálogo comentado de las especies de la sección Brevispicae presentes en el territorio Boliviano.

\section{MATERIALES Y MÉTODOS}

Se revisaron cerca de 140 ejemplares depositados en los herbarios: BOLV, LPB y USZ, abreviados de acuerdo con Thiers (2011). Dichos especímenes se estudiaron empleando las técnicas utilizadas en los tratamientos taxonómicos clásicos, la terminología botánica empleada está dada de acuerdo con Font Quer (2001). Los especímenes fueron delimitados siguiendo el concepto morfológico de especie (Davis \& Heywood 1963, Crisci
1994, Uribe-Meléndez 2008), en el que se define a las especies "como un conjunto de individuos que presentan un espectro continuo de variación fenotípica y está separado de otros conjuntos por discontinuidades morfológicas. Para la identificación de los especímenes se emplearon las claves y descripciones presentadas en Smith $(1954,1965)$, Alfaro (1981), Steyermark (1988), Smith \& Steyermark (1998), Palacios-Duque \& Fernández-Alonso (2005), PalaciosDuque (2009), Sampaio (2009), Vásquez (2009) y Sampaio \& Souza (2011). La información sobre el material tipo y la nomenclatura de las especies se obtuvo de la literatura especializada en el grupo (Smith 1954, Alfaro 1981, Steyermark 1988, Sampaio 2009), y la consulta de las bases de datos www.ipni.org; http://plants.jstor.org y de los herbarios A, K, MO, NY y US abreviados de acuerdo con Thiers (2011).

\section{RESULTADOS}

Para Bolivia se reconocen siete taxones de la sección Brevispicae: S. eichlerii K. Schum., S. guianensis (Aubl.) Benth., S. obtusifolia (Moric.) K. Schum., S. picapica Standl., S. pubescens (Poepp. \& Endl.) K Schum., S. spathulata Earle Sm. y S. tuerckheimii D. Donn.). Se provee una clave para identificar las especies, la cual, está basada tanto en caracteres reproductivos como vegetativos. Adicionalmente se entregan aspectos relacionados con la distribución, hábitat, afinidades u observaciones taxonómicas, nombres comunes y usos locales.

Clave para separar los subgéneros Quadrisepala y SLOANEA y las Secciones del Subgénero SLOANEA

1. Estípulas rápidamente caedizas con el desarrollo de las hojas. Inflorescencias racemosas o cimosas, laterales o raramente terminales. Cáliz con 4 sépalos (raramente 5), de igual forma y tamaño, que recubren los verticilos reproductivos antes de la

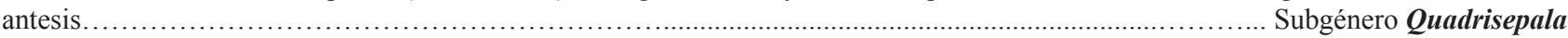

1'. Estípulas tardíamente caedizas o persistentes con el desarrollo de las hojas. Inflorescencias racemosas laterales. Cáliz con 4-11sépalos, desiguales en forma y tamaño, que no cubren los verticilos reproductivos antes de la antesis.......................... Subgénero Sloanea (2)

2. Estípulas grandes, desde 1,5 hasta $15 \mathrm{~cm}$ de longitud, con nervaduras de primer o segundo orden, persistentes a lo largo de las ramitas. Racimos florales esparcidos, desde pequeños a grandes. Cápsulas de 2,5 hasta $10 \mathrm{~cm}$ de longitud; espinas dimórficas o monomórficas, largas (más de $2 \mathrm{~cm}$ ), rectas o recurvadas, rígidas, no irritantes; 1-4 semillas Sección Sloanea

$2^{\prime}$. Estípulas pequeñas, sin superar $1 \mathrm{~cm}$ de longitud, sin nervaduras, rápidamente caedizas (sólo apreciables al final de las ramitas). Racimos florales agrupados, generalmente pequeños. Cápsulas de 1,0 hasta $3,5 \mathrm{~cm}$ de longitud, generalmente con menos de 2,5 cm; espinas monomórficas, cortas (menos de 1,5 cm), rectas, flexibles, algunas veces irritantes; 1 a más de 10 semillas. Sección Brevispicae

\section{TRATAMIENTO TAXONÓMICO}

Subgénero Sloanea Earle Sm. Contr. Gray Herb. 175: 46. 1954.

Grandes árboles o raramente arbustos. Estípulas presentes con el desarrollo de las hojas, lineares, lanceoladas o foliáceas. Inflorescencias racemosas, axilares. Sépalos
4-11, desiguales en forma y tamaño, sin llegar a cubrir los estambres y el gineceo. Cápsulas provistas de espinas cortas o largas y flexibles, gruesas o delgadas, algunas veces irritantes.

Especie tipo: Sloanea dentata L. Lectotipo designado por N. L. Britton \& P. Wilson, Scient. Surv. Porto Rico 5: 541 (1924). 
Gayana Bot. 70(2), 2013

Sección Brevispicae Earle Sm., Contr. Gray Herb. 175: 46. 1954.

Brachystachyae Benth., Biol. J. Linn. Soc. 5: Suppl. 63. 1861. Pro parte.

Árboles o raramente arbustos. Estípulas pequeñas, principalmente deltoides o lanceoladas, rápidamente caedizas, sin nervaduras. Inflorescencias axilares, racemosas, generalmente agrupadas. Sépalos 4-11, desiguales en forma y tamaño. Antera generalmente de igual tamaño que el filamento. Cápsulas de hasta $3,5 \mathrm{~cm}$ de longitud, aunque generalmente son menores de $2,5 \mathrm{~cm}$ de longitud, provistas de espinas cortas y flexibles (inermes en $S$. granulosa Ducke). Especie tipo: Sloanea massoni Swartz.

\section{Clave para reconocer las especies de la Sección BreVispicae en Bolivia}

1. Láminas foliares discoloras cuando secas; envés densamente pubescente (toda la superficie)

1'. Láminas foliares de un solo color cuando secas (generalmente verdoso o café); envés glabro o con indumento escaso sobre las nervaduras.

2. Estípulas de 0,7-1,5 cm de largo, con nervio medio; pecíolo con una línea tricomas en la superficie adaxial; haz castaño, envés ligeramente glauco, indumento escaso. Cápsulas maduras de hasta $2,5 \mathrm{~cm}$ de largo; espinas brevemente pubescentes

7. S. tuerckheimii

2'. Estípulas con menos de $0,5 \mathrm{~cm}$ de largo, sin nervio medio; pecíolo sin una línea de tricomas en la superficie adaxial; haz café brillante, envés glauco, indumento abundante. Cápsulas maduras de hasta $1,5 \mathrm{~cm}$ de largo; espinas densamente pubescentes

1. S. eichleri

3. Inflorescencias de 1-7 cm de largo; flores con cinco a once sépalos de tamaño y forma variable. Cápsulas con espinas agrupadas, difícil a ligeramente desprendibles

3'. Inflorescencias de 1-3 cm de largo; flores con cuatro sépalos de igual forma y tamaño. Cápsulas con espinas muy esparcidas y fácilmente desprendibles

3. S. obtusifolia

4. Hojas opuestas, láminas obovadas o ampliamente elípticas de hasta $25 \mathrm{~cm}$ de largo; ápice redondeado a brevemente cuspidado; espinas capsulares densamente agrupadas.

4'. Hojas alternas, láminas elípticas de hasta $7 \mathrm{~cm}$ de largo; ápice largamente acuminado; espinas capsulares laxamente esparcidas. 4. S. picapica

5. Pecíolos negruzcos (pulvínulos más oscuros), glabros; lámina obovada, glabra. Cápsulas de 1-1,3 cm de largo, espinas rectas y amarillas. 2. S. guianensis 5'. Pecíolos y pulvínulos castaños, pubérulos, lámina obovada o ampliamente elípticas, pubescentes en el envés. Cápsulas de 1,5-2 cm de largo; espinas cónicas castañas.

6. Hojas agrupadas hacia el final de las ramitas; pecíolos de $0,5-0,8 \mathrm{~cm}$ de longitud; laminas obovadas, generalmente de color castaño cuando secas; envés pubérulo a glabro. Cápsulas con la superficie interna de color castaño oscuro.......

..6. S. spathulata

6'. Hojas esparcidas sobre las ramitas; pecíolos de 1-5 cm de longitud; láminas ampliamente elípticas, generalmente verduzcas cuando secas; envés pubérulo (principalmente en las nervaduras). Cápsulas con la superficie interna blanquecina. .... 5. S. pubescens

1. Sloanea eichleri K. Schum., in Martius, Fl. Bras. 12(3): 183, pl. 28, f. 1. 1886. TIPO: Brasil. Rio Tocantins, Weddel 2478 (Lectotipo designado por Sampaio (2009), B no visto, Isolectotipo P no visto).

Sloanea obidensis Ducke, Arch. Jard. Bot. Rio de Janeiro. 2: 160. 1935. TIPO: Brasil: Para: Obidos, 11-XI-1919 (fl), Ducke s.n., Herb. Jard. Bot. Rio de Janeiro 12369 (Lectotipo designado por Sampaio (2009), RB!, Isolectotipos GH no visto, K no visto, RB!, US!).

DESCRIPCIÓN: El material estudiado concuerda perfectamente con las descripciones presentadas en Alfaro (1981), Palacios-Duque (2009), Sampaio (2009).

DistribuCión y háBITAT: Se ha documentado en Brasil, Colombia, Ecuador, Guayana Francesa, Perú, Venezuela, de acuerdo con Smith (1954), Alfaro (1981), Liesner (1993),
Jaramillo (1999), Palacios-Duque (2009), Vázquez (2009), Sampaio (2009), Sampaio (2010). Se encuentra en bosques de tierra firme, con suelos tipo ultisoles, en los cuales se ha reportado asociada con especies de las familias Arecaceae, Bignoniaceae, Fabaceae, Lecythidaceae, Moraceae y Sapotaceae.

NomBre común: En la lengua chácabo se conoce como "xahuamachi" Boom 4134, 4522 y en Español se conoce como "urucucillo" Balcazar \& Oliver 1387.

Usos: Se menciona su uso en la medicina tradicional para tratar infecciones en los ojos, Boom 4134. De igual manera, su madera es empleada como combustible vegetal, Boom 4522 y Balcazar \& Oliver 1387.

Observaciones: Se distingue por el abundante y fino indumento (blanquecino-castaño claro) que poseen sus 
hojas en envés y por lo cual, las hojas presentan un aspecto discoloro cuando secas (Fig. 1A). Se relaciona con Sloanea sinemariensis Aubl., no obstante, esta última presenta menos indumento y flores con 5-6 sépalos (siendo uno más pequeño que los demás), mientras que en $S$. eichleri las flores poseen 5-9 sépalos todos variables en su forma y tamaño. S. eichleri después del secado presenta hojas discoloras y por ello frecuentemente sus especímenes son identificados como Sloanea tuerckheimii Donn. Smith., véase las observaciones de esta última especie.

Material examinado: BOLIVIA. Beni, Ballivian, Yacuna, el Mirador, 26-IX-1991 (fl), Beck 20028 (LPB); Vaca Diez, vecindad de Chácobo, $11^{\circ} 45^{\prime} \mathrm{S}, 66^{\circ} 02^{\prime} \mathrm{W}, 200 \mathrm{~m}, 11-\mathrm{XII}-$ 1983 (fr), Boom 4134 (LPB), 21-II-1984 (fr), Boom 4522 (LPB); Barraca Monte Livano, 18-VIII-1999, (st.), Balcazar \& Oliver 1387 (USZ). La Paz, Abel Ituralde, Ixiamas, Arroyo Seco, $13^{\circ} 35^{\prime} \mathrm{S}, 68^{\circ} 28^{\prime} \mathrm{W}, 299$ m, 20-I-2003 (fr), Balcazar et al. 2724 (LPB, USZ); rio Saturiapo, 135' $\mathrm{S}$, $68^{\circ} 15^{\prime} \mathrm{W}, 620-650 \mathrm{~m}, 2-\mathrm{VI}-1990$ (fr), Gentry \& Foster 70865 (LPB). Pando, Manuripi, 11 ${ }^{\circ} 44^{\prime} 34^{\prime \prime} \mathrm{S}, 67^{\circ} 59^{\prime} 00^{\prime \prime} \mathrm{W}$, 200 m, 16-III-1994 (fr), Jardim et al. 437 (BOLV, LPB, USZ). Santa Cruz, Velasco, campamento Toledo, $160 \mathrm{~m}$, 7-V-1994 (fr), Guillen \& Choré 1732 (BOLV, USZ); San Ignacio de Velasco, $14^{\circ} 35^{\prime} 37^{\prime \prime} \mathrm{S}, 61^{\circ} 10^{\prime} 45^{\prime \prime} \mathrm{W}, 200 \mathrm{~m}$, 7-III-2005 (st.), Toledo et al. 1802 (USZ); Estación Flor de Oro, cerca al río Itenez, $13^{\circ} 30^{\prime} \mathrm{S}, 61^{\circ} 00^{\prime} \mathrm{W}, 260 \mathrm{~m}, 25$-III1992 (st.), Perry \& Cuellar 818 (USZ); Reserva ecológica El Refugio, $14^{\circ} 41^{\prime} 27^{\prime \prime} \mathrm{S}, 61^{\circ} 10^{\prime} 14.7^{\prime \prime} \mathrm{W}, 200 \mathrm{~m}, 15-\mathrm{V}-1995$ (fr), Guillen \& Choré 3658 (LPB); Reserva ecológica El Refugio, $14^{\circ} 42^{\prime} 30^{\prime \prime} \mathrm{S}, 61^{\circ} 10^{\prime} 29.8^{\prime \prime} \mathrm{W}, 180 \mathrm{~m}, 12-\mathrm{V}-1995$ (st.), Guillen 3618 (USZ); Parque Nacional Noel Kempff, campamento La Torre, $13^{\circ} 39^{\prime} \mathrm{S}, 60^{\circ} 49^{\prime} \mathrm{W}, 200 \mathrm{~m}, 18 \mathrm{-V}-$ 1994 (fr), Quevedo et al. 2595 (LPB, USZ); campamento Los Fierros, 14³3'29' 'S, 6055'41' 'W, 200 m, 4-VII-1996 (fr), Jardim \& Foster 3039 (USZ); campamento Los Fierros, $14^{\circ} 33^{\prime} 30^{\prime \prime} \mathrm{S}, 60^{\circ} 49^{\prime} 12^{\prime \prime} \mathrm{W}, 155 \mathrm{~m}, 3-\mathrm{XI}-1993$ (fl. pas.ant.), Quevedo et al. 2437 (USZ).

2. Sloanea guianensis (Aubl.) Benth., Biol. J. Linn. Soc. 5: Suppl. 69. 1861.

Basiónimo: Ablania guianensis Aubl., Hist. Pl. Guiane. 1: 585-587, t. 234. 1775. TIPO: Guiana Francesa, "Sinemarientibus", Aublet sin número (Lectotipo designado por Sampaio (2009), BM 795143 no visto).

DESCRIPCIÓN: El material estudiado concuerda perfectamente con las descripciones presentadas en Alfaro (1981), Palacios-Duque (2009), Sampaio (2009).

Sinonimia: Se recomienda ver Smith (1954), Sampio (2009).

Distribución Y HÁBITAT: Se ha documentado desde Costa
Rica hasta Brasil (Smith 1954). Se encuentra en bosques de tierra firme con fuertes pendientes, con suelos tipo oxisoles, rojos y derivados de areniscas. Reportándose asociada con algunas especies de los géneros Astrocaryum, Iriartea (Arecaceae), Bertholletia, Couratari (Lecythidaceae) y Spondias (Anacardiaceae).

Nombres comunes: En la lengua chácobo se conoce como “xahuamachi” Bergeron 931. En español es conocida como "coloradillo, gabetillo y urucusillo" Moraes 1033, Neill \& Quevedo 9446, Killen et al. 2939, Kuno 343, Meneces \& Terceros 206, 207, 342, Llanque et al. 900 y Rivero 142.

Usos: La madera de sus bambas o aletones es empleada para la fabricación de cabos para hachas Neill \& Quevedo 9446 y la del tronco y ramas es empleada como combustible o leña Rivero 142 (LPB).

OBSERVACIONES: Esta especie se reconoce principalmente por sus hojas opuestas con margen ondulado o algunas veces dentado y sus cápsulas pequeñas con abundantes espinas de color verduzco a amarillento (Fig. $1 \mathrm{~B}, \mathrm{C}$ ).

Material examinado: BOLIVIA. Beni, Ballivian, Escuela Técnica Agrícola Río Colorado, $14^{\circ} 55^{\prime} \mathrm{S}, 67^{\circ} 05^{\prime} \mathrm{W} 350$ m, 28-29-VI-1991(fr), Killen et al. 2939 (BOLV); Beni, Ballivian, estación biológica Beni, $14^{\circ} 44^{\prime} \mathrm{S}, 66^{\circ} 20^{\prime} \mathrm{W} 250$ m, 6-XI-1985 (fr), Solomon 14604 (LPB); Beni, Ballivian, Serrania Pilón Lajas, $1^{\circ} 15^{\prime} \mathrm{S}, 67^{\circ} 00^{\prime} \mathrm{W}$ 850-900 m, 3-7-V-1991(fr), Killeen et al. 3091 (BOLV); Beni, Ballivian, Triunfo, 240 m, 24-VIII-1976 (fl), Cruz \& Terceros 206 (BOLV, LPB); Beni, Ballivian, Triunfo, $240 \mathrm{~m}, 29-\mathrm{VIII}-$ 1976 (fl), Meneces \& Terceros 207 (BOLV); Beni, Ballivian, San Borja a Trinidad, 226 m, 25-IX-1976 (fr), Meneces \& Terceros 342 (BOLV); Beni, Marban, Trinidad $164 \mathrm{~km}$ hacia Santa Cruz, pasando San Pablo, 28-VII-1985 (fr), Beck 12225 (LPB); Beni, Marban, Trinidad 164 km hacia Santa Cruz, pasando San Pablo, 28-VII-1985 (fl), Beck 12223 (LPB); Beni, Rurrenabaque, X-1921 (fr), Mulford 1265 (BOLV); Beni, Vaca Diez, Alto Ivón, $11^{\circ} 45^{\prime} \mathrm{S}, 66^{\circ} 02^{\prime} \mathrm{W} 200$ m, X-1993 (fl), Bergeron 931 (LPB); Beni, Yacuma, estación biológica Beni, $14^{\circ} 39^{\prime} \mathrm{S}, 66^{\circ} 22^{\prime} \mathrm{W}, 250 \mathrm{~m}, 1-\mathrm{I}-1988$ (fr), Moraes 1033 (LPB); Beni, Yacuma, estación biológica Beni, Río Maniqui, El Chacal, 200 m, 18-VIII-1991 (fr), Palacios 7557 (LPB); Beni, Yacuma, estación biológica Beni, 14³0'S, 66³7'W, 200 m, 18-VIII-1994 (fr), Rivero 142 (LPB); Beni, Yacuma, $15^{\circ} 10^{\prime} \mathrm{S}, 66^{\circ} 28^{\prime} \mathrm{W} 250 \mathrm{~m}, 23-\mathrm{XI}-1988$ (fr), Foster

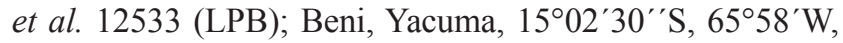
1-XII-1991 (fr), Llanque et al. 900 (LPB). Cochabamba, Carrasco, Estación Valle del Sajta, $17^{\circ} 20^{\prime} \mathrm{S}, 60^{\circ} 50^{\prime} \mathrm{W}, 29-$ X-1991 (st), Galarza et al. 24 (BOLV). Pando, Manuripi, $11^{\circ} 35^{\prime} 39^{\prime \prime} \mathrm{S}, 68^{\circ} 02^{\prime} 52^{\prime \prime} \mathrm{W} 180$ m, 9-IX-1995 (fl), Jardim 2402 (BOLV, LPB, USZ). La Paz, Abel Iturralde, delta del arroyo Arana y el río Tuichi, $14^{\circ} 27^{\prime} \mathrm{S}, 67^{\circ} 46^{\prime} \mathrm{W} 350 \mathrm{~m}, 21-$ 
VI-1995 (fl), Kuno 343 (LPB). Santa Cruz, Ichilo, rio Ibabo, Reserva forestal Choré, campamento perdido, $16^{\circ} 32^{\prime} \mathrm{S}$,

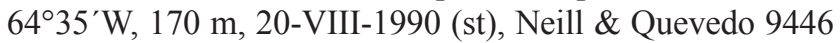
(LPB); Santa Cruz, Parque Nacional Noel Kempff M., campamento los Fierros, $14^{\circ} 48^{\prime} 41^{\prime \prime} \mathrm{S}, 60^{\circ} 23^{\prime} 45^{\prime \prime} \mathrm{W}, 225 \mathrm{~m}$, 17-X-1993 (fr), Killeen et al. 5625 (BOLV, LPB, USZ).

3. Sloanea obtusifolia (Moric.) K. Schum., in Martius, Fl. Bras. 12(3): 181. 1886.

Basiónimo: Adenobasium obtusifolium Moric., Pl. Nouv. 83-84, t. 55. 1840. TIPO: Brasil. Bahia, Blanchet 1659 (Holotipo K!, Isotipo F!).

Sloanea fernando-costae Hoehne., Arq. Bot. Estado Sao Paulo 1: 93, t 96. 1942. TIPO: Brasil. Sao Paulo: Jardin Botánico de Sao Paulo, 21-VIII-1933, Planta viva 324, O. Handro 30844 (Holotipo SP!, Isotipos K!, RB!, GH no visto).

DESCRIPCIÓN: El material estudiado concuerda perfectamente con la descripción presentada en Sampaio (2009).

DistribuCión Y hábitat: Se ha registrado en Brasil, Perú y Venezuela (Smith 1954). Muestra preferencia por bosques primarios no inundables, en los que se encuentra asociada con algunas especies de los géneros Eschweilera Mart. ex DC. (Lecythidaceae), Iriartea Ruiz \& Pav. (Arecaceae) y Terminalia L. (Combretaceae).

NomBre COMÚN: "urucucillo" Zárate \& Zurita 2764 (LPB).

ObSERVACiOnes: Por el aspecto general de su follaje y hábito se relaciona con S. guianensis Aubl., sólo diferenciándose de ella por poseer inflorescencias menores de $4 \mathrm{~cm}$ y por sus flores con 4 sépalos y las espinas de la cápsula que generalmente se encuentran más separadas (Fig. 1D).

Material examinado: BOLIVIA. Cochabamba, Carrasco, Estación experimental Valle de Sajta, $17^{\circ} 00^{\prime} \mathrm{S}, 64^{\circ} 46^{\prime} \mathrm{W}$, 290 m, 00-IX-1991 (fl), Pariona \& Quevedo 1240 (LPB); Cochabamba, Carrasco, Valle de Sajta, $17^{\circ} 05^{\prime} 18^{\prime \prime} \mathrm{S}$, 644ㅇ'06' 'W, 390 m, 22-X-2007 (fr), Zárate \& Zurita 2764 (LPB); Valle de Sajta, $17^{\circ} 05^{\prime} 15^{\prime \prime} \mathrm{S}, 64^{\circ} 46^{\prime} 01^{\prime \prime} \mathrm{W}, 390 \mathrm{~m}$, 4-I-2008 (fr), Zárate \& Villca 2802 (LPB); La Paz, Sud Yungas, Alto Beni, Serranía de los Marimonos, 610 m, 24IV-1993 (fr), Seidel \& Vaquiata 7264 (LPB).

4. Sloanea picapica Standl., Publ. Field Mus. Nat. Hist., Bot. Ser. 22: 89. 1940. TIPO: HONDURAS: Colón, Guarunta: Wisperinini Camp. Wolfgang von Hagen \& Christine von Hagen 1390 (Holotipo: F!; Isotipos: NY!, F no visto).
DESCRIPCIÓN: El material estudiado concuerda perfectamente con las descripciones presentadas en Smith (2001), PalaciosDuque \& Fernández-Alonso (2007), Palacios-Duque (2009).

DistribuCión y hÁBITAT: Se registra para Colombia, Costa Rica, Nicaragua y Panamá Smith (1954), Smith (1965), D'Arcy (1987), Smith (2001), Palacios-Duque \& FernándezAlonso (2007), Palacios-Duque (2009). Se ha encontrado en bosques primarios de tierras altas con fuertes pendientes, en los que predominan Phytelephas macrocarpa Ruiz \& Pav., (Arecaceae) y Pseudolmedia laevis (Ruiz \& Pav.) J. F. Macbr. (Moraceae).

NOMBRE COMÚN: En español es conocida como "urucusillo" y en la lengua yuracare se conoce como "muni" y "beymishtu" Thomas \& Berdeja 1542.

ObSERVACIONES: Esta especie se reconoce por sus hojas pequeñas, elípticas, largamente acuminadas con filotaxia alterna a subopuesta y principalmente por sus frutos con espinas cónicas dispersas (Fig. 2A).

Material examinado: BOliViA. Cochabamba, Chapare, $16^{\circ} 23^{\prime} \mathrm{S}, 65^{\circ} 57^{\prime} \mathrm{W}, 230 \mathrm{~m}, 4-\mathrm{IV}-2005$ (fr), Thomas \& Berdeja 1542 (LPB). La Paz, Iturralde, Parque NacionalANMI- Madidi, $14^{\circ} 20^{\prime} 52^{\prime \prime} \mathrm{S}, 67^{\circ} 58^{\prime} 50^{\prime \prime} \mathrm{W}, 361 \mathrm{~m}, 16-\mathrm{X}-$ 2002 (st.), Cabrera et al. 166 (LPB).

5. Sloanea pubescens Benth., Biol. J. Linn. Soc. 5 (Suppl. 2): 69. 1861. TIPO: Brasil. Amazonas, Ega, Poeppig 2662 (Lectotipo designado por Sampaio (2009), W no visto, Isolectotipos $\mathrm{F}$ !, $\mathrm{GH}, \mathrm{W}$ no vistos).

Sloanea xylocarpa Rusby, Mem. New York Bot. Gard. 7: 294. 1927. TIPO: Bolivia. Tumpassa, M. Cardenas 1979 (Holotipo NY!, Isotipos US!, K, GH no vistos,).

Sloanea pseudodentata Ducke. Arch. Jard. Bot. Rio de Janeiro. 2: 159. 1935. TIPO: Brasil. Para, Rio Tapajos, RB 18378, Ducke s. n. (Lectotipo designado por Sampaio (2009), RB no visto, Isolectotipo K!, US no visto).

DESCRIPCIÓN: El material estudiado concuerda perfectamente con las descripciones presentadas en Alfaro (1981), Sampaio 2009).

DistribuCiÓn y hÁBITAT: Se conoce en Ecuador, Brasil, Surinam y Venezuela Smith (1954), Alfaro (1981), Sampaio (2009, 2010), Vázquez (2009). Se ha reportado en bosques de tierras firmes ubicados entre los 330-1.468 m, con fuertes pendientes, en donde predominan suelos tipo ultisoles arcillosos y rojizos, derivados areniscas, en donde se asocia con Dictyocaryum lamarckianum (Mart.) H. Wendl., y Socratea exorrhiza (Mart.) H. Wendl. (Arecaceae). 

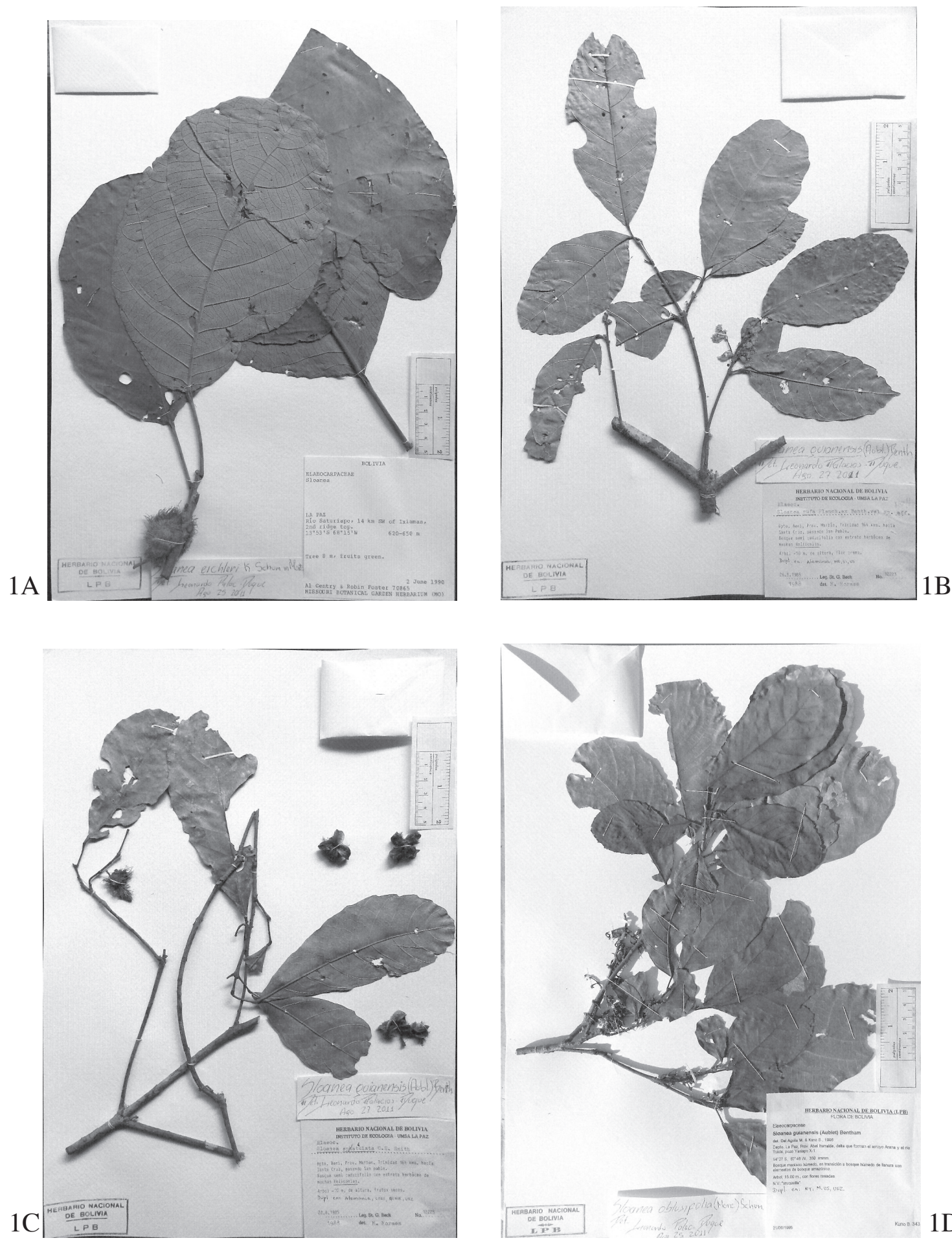

Figura 1. A. Sloanea eichleri K. Schum. Detalle de una ramita fructífera. Gentry \& Foster 70865 (LPB). B. Sloanea guianensis (Aubl.) Benth. Detalle de una ramita florífera. Beck 12223 (LPB). C. Sloanea guianensis (Aubl.) Benth. Detalle de una ramita fructífera. Beck 12225 (LPB). D. Sloanea obtusifolia (Morich.) K. Schum. Detalle de una ramita florífera. Kuno 343 (LPB).

Figure 1. A. Sloanea eichleri K. Schum. Details of a branch and its fruits. Gentry \& Foster 70865 (LPB). B. Sloanea guianensis (Aubl.) Benth. Details of a branch and its flowers. Beck 12223 (LPB). C. Sloanea guianensis (Aubl.) Benth. Details of a branch and its fruits. Beck 12225 (LPB). D. Sloanea obtusifolia (Morich.) K. Schum. Details of a branch and its flowers. Kuno 343 (LPB). 
$2 \mathrm{~A}$
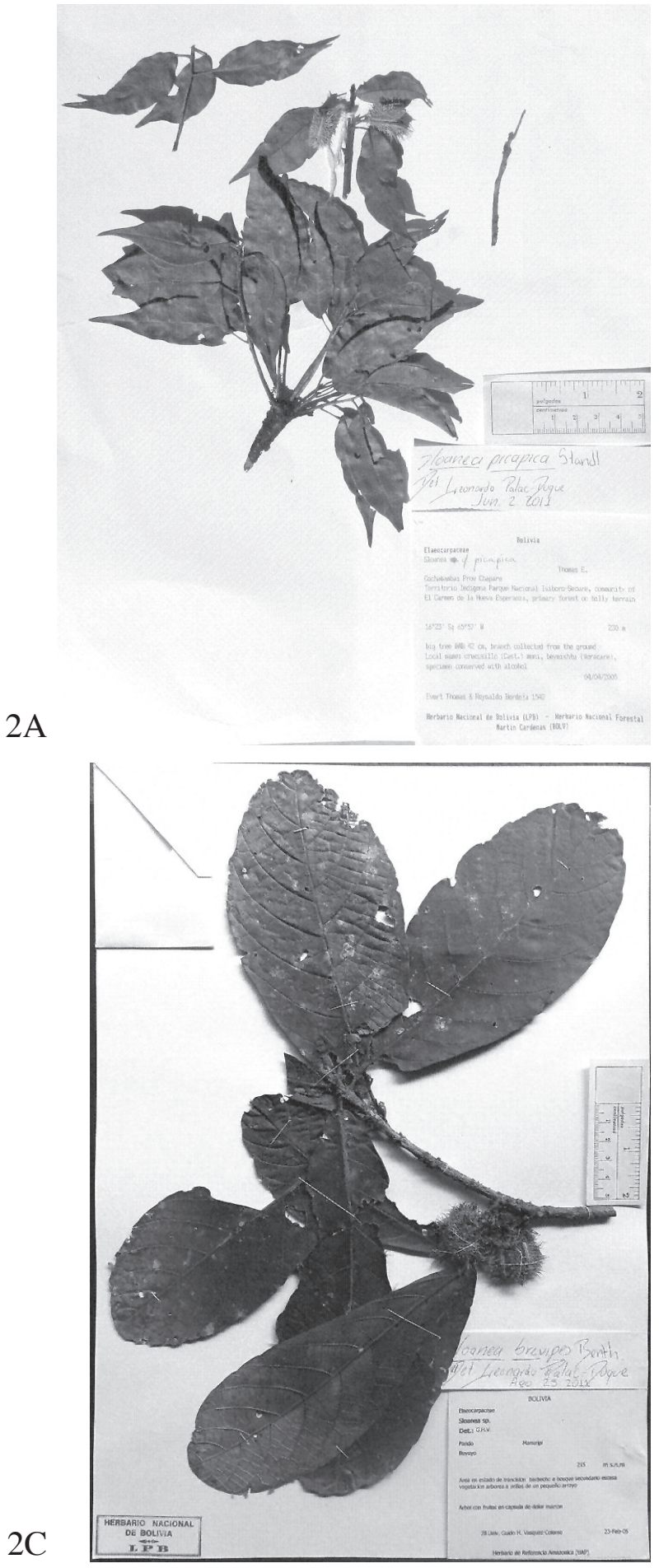

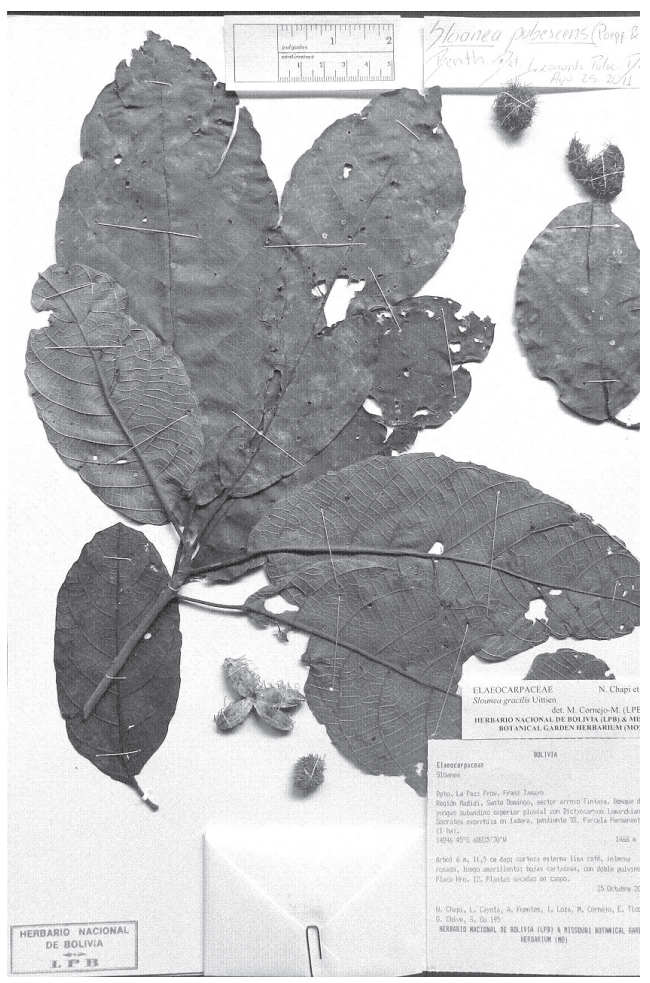

$2 \mathrm{~B}$

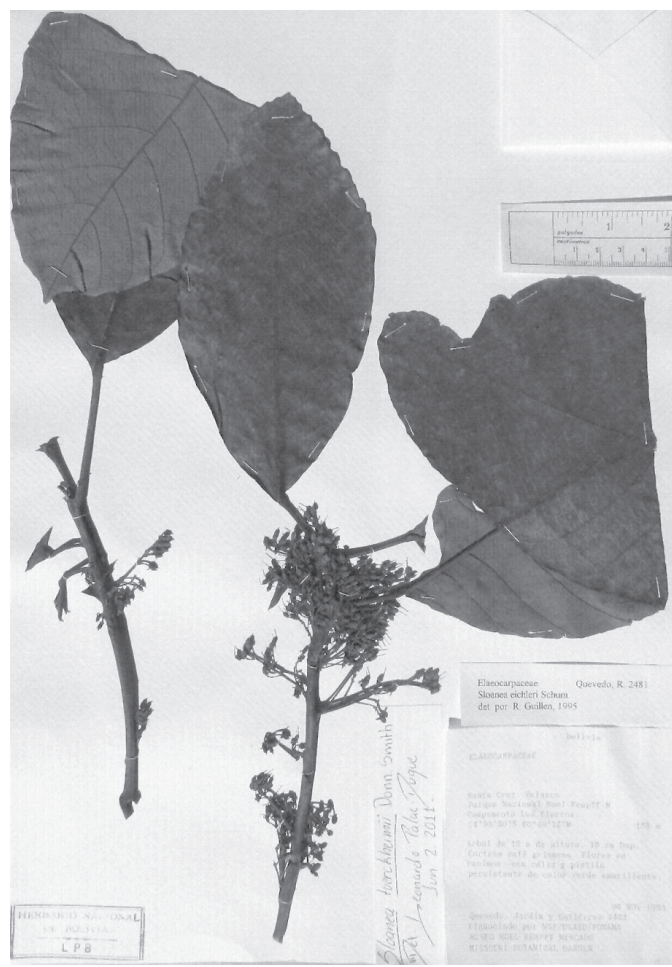

2D

Figura 2. A. Sloanea picapica Stand. Detalle de una ramita fructífera. Thomas \& Berdeja 1542 (LPB). B. Sloanea pubescens (Poepp. \& Endl.) Benth. Detalle de una ramita y frutos. Chapi et al. 195 (LPB). C. Sloanea spathulata Earle Sm. Detalle de una ramita fructífera. Vásquez 78 (LPB). D. Sloanea tuerckheimii D. Smith. Detalle de una ramita florífera (flores pasada la antesis). Quevedo et al. 2481 (LPB).

Figure 2. A. Sloanea picapica Stand. Details of a branch and its fruits. Thomas \& Berdeja 1542 (LPB). B. Sloanea pubescens (Poepp. \& End1.) Benth. Details of a branch and its fruits. Chapi et al. 195 (LPB). C. Sloanea spathulata Earle Sm. Details of a branch and its fruits. Vásquez 78 (LPB). D. Sloanea tuerckheimii D. Smith. Details of a branch and its flowers (flowers after anthesis). Quevedo et al. 2481 (LPB). 
NOMBRE COMÚN: “urucusillo” Killen et al. 3125.

ObSERVACIONes: Esta especie se distingue por sus hojas opuestas, esparcidas sobre las ramitas y provistas de un indumento abundante en el envés, de igual manera se reconoce por la coloración blanca de sus cápsulas en la superficie interna (Fig. 2B).

Materiales examinados: BOliVia. Beni, Ballivian, Serranía Pilon Lajas, $15^{\circ} 15^{\prime} \mathrm{S}, 67^{\circ} 00^{\prime} \mathrm{W}, 850-900 \mathrm{~m}, 3-7-$ V-1991 (st), Killen et al. 3125 (BOLV). Cochabamba, Villa Tunari a Puerto Patiño, 320 m, 19-X-1977 (fr), Meneces \& Harsthorn 2048 (BOLV); Chapare, Villa Fátima, $18^{\circ} 28^{\prime} 37^{\prime \prime} \mathrm{S}, 68^{\circ} 53^{\prime} 88^{\prime \prime} \mathrm{W} 280 \mathrm{~m}, 1$-XII-2004 (fr), Villarroel et al. 10 (USZ). La Paz, Franz Tamayo, Región

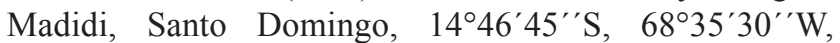
1468 m, 25-X-2006 (fr), Chapi et al. 195 (LPB); Sud Yungas, Alto Beni, $600 \mathrm{~m}, 16-\mathrm{XI}-1991$ (fr), Seidel et al. 5637 (LPB); Sud Yungas, Alto Beni, 600 m, 14-XI1991 (fr), Seidel et al. 5565 (LPB). Santa Cruz, Velasco, Campamento El Refugio, $14^{\circ} 45^{\prime} \mathrm{S}, 61^{\circ} 01^{\prime} \mathrm{W} 180 \mathrm{~m}$, 2-VI1994 (fr), Guillen \& Chore 1663 (USZ). Campamento Los Fierros, 14 33'30' 'S, 60 49'12' W 155m, 5-XI-1993 (fr), Quevedo et al. 2457 (USZ).

6. Sloanea spathulata Earle Sm., Contr. Gray. Herb. 175: 43. 1954. TIPO: Brasil: Estado de Acre, cerca de la desembocadura del rio Macauhan (afluente del rio Yaco), 8-VIII-1933, Krukoff 5337 (Holotipo: A no visto; Isotipos: BM!, F!, K!, MICH!, MO!, NY!, SP!, U!, US!).

DESCRIPCIÓN: El material estudiado concuerda perfectamente con las descripciones presentadas en Smith (1954), Alfaro (1981).

DisTRIBUCIÓN Y HÁBITAT: Se ha registrado para Brasil, Ecuador, Perú y Venezuela Smith (1954), Liesner (1993), Jaramillo (1999), Vázquez (2009). En Bolivia se ha encontrado en bosques con fuertes pendientes, sobre suelos rojos arcillosos derivados de rocas areniscas y ocasionalmente ha sido colectada en bosques estacionalmente inundables o en cercanías de arroyos. Se ha reportado asociada con especies de las familias Annonaceae, Lauraceae y Moraceae.

NOMBRE COMÚN: En la lengua yuracare es conocida como "beymishtu" Thomas \& Berdeja 1457 (LPB).

ObSeRvaCiones: Se distingue por sus hojas opuestas, obovadas a espatuladas, ligeramente agrupadas hacia el final de las ramitas. Las flores presentan 5-6 sépalos pubescentes en la superficie externa y glabros en la interna; los filamentos son pubescentes, el conectivo estrecho, de color claro, anteras glabras con una pequeña arista. Cápsulas elípticas a ovoides, densamente cubierta por espinas escasamente pubescentes (Fig. 2C). Por sus hojas espatuladas y cortos pecíolos S. spathulata se relaciona con Sloanea brevipes Benth., no obstante se separan claramente por presentar esta última especie las hojas claramente agrupadas al final de las ramitas. Flores con 6-8 sépalos, conectivo amplio y de color oscuro y las espinas capsulares que son densamente pubescentes.

Material examinado: BOLIVIA. Beni, Ballivian, Serranía Pilón Lajas, $15^{\circ} 15^{\prime} \mathrm{S}, 67^{\circ} 00^{\prime} \mathrm{W}, 850-900 \mathrm{~m}, 3-7-\mathrm{V}-1991$ (st.), Killen et al. 3075 (BOLV). Cochabamba, Chapare, $16^{\circ}$ $24^{\prime} \mathrm{S}, 65^{\circ} 54^{\prime} \mathrm{W}, 240 \mathrm{~m}, 22-\mathrm{XI}-2004$ (fr), Thomas \& Berdeja 1457 (LPB); Valle de Sacta $17^{\circ} 00^{\prime} \mathrm{S}, 64^{\circ} 46^{\prime} \mathrm{W}, 290 \mathrm{~m}, 8-9-$ VII-1989 (fr), Smith et al. 13640 (BOLV), Cochabamba, Carrasco, Valle de Sacta, $17^{\circ} 05^{\prime} 12^{\prime \prime} \mathrm{S}, 64^{\circ} 46^{\prime} \mathrm{W}, 390 \mathrm{~m}$, 5-X-2007 (fr), Zárate \& Vargas 2627 (BOLV). La Paz, Franz Tamayo, Parque Nacional Madidi, río Hondo, Arroyo Negro, 14 39' $12^{\prime \prime}$ S, $67^{\circ} 48^{\prime} 7^{\prime \prime} \mathrm{W}, 340$ m, 27-III-2002 (st.), Seidel et al. 8965 (LPB). Pando, Manuripi, Buyuyo, 215 m, 23-II-2005 (fr), Vásquez 78 (LPB).

7. Sloanea tuerckheimii Donn. Sm. Bot. Gaz. 57 (5): 416. 1914. TIPO: Guatemala: Alta Verapaz: in silvis ad Cubilquitz, alt. 350 m, Mart et Maj. 1913, H. von Tuerckheim 4157 (Holotipo B!, Isotipos CORD!, ILL!, S!, U!).

Sloanea macrophylla Benth. ex Turcz., Bull. Soc. Imp. Naturalistes Moscou 31(1): 224. 1858. TIPO: Brazil: Amazonas: Prope Panure ad Rio Vaupes; IX-1852-I-1853; Spruce 2478 (BR!, F!, K!, MPU!, NY!, TCD!, S!, G, GH no vistos). Pro parte.

Sloanea usurpatrix Sprague \& L. Riley, Bull. Misc. Inform. Kew 1924 (1): 20. 1924. TIPO: British Guiana, Schomburgk 1021 (Holotipo K!, Isotipo F!). Pro parte.

Sloanea eriostemon Sprague \& L. Riley, Bull. Misc. Inform. Kew 1924(1): 19. 1924. TIPO: British Honduras: Forest near Manatee Lagoon May 1913; M.E. Peck 400 (Holotipo: $\mathrm{K}$ !; Isotipo NY!).

DESCRIPCIÓN: El material estudiado concuerda perfectamente con las descripciones presentadas en Alfaro (1981), Smith (2001), Sampaio (2009).

DistribuCIÓN Y hÁBITAT: Se encuentra desde Nicaragua hasta Brasil Smith (1954), Palacios-Duque (2009), Vázquez (2009). En Bolivia se ha encontrado creciendo en bosques estacionalmente inundados y creciendo en asocio con algunas especies de las familias Arecaceae, Lauraceae y Lecythidaceae.

Nombres comunes: "cabeza de mono, chocolatillo, urococillo y urucucillo" Zárate \& Vargas 2557, Zárate \& 
Zurita 2767, Zárate \& Villca 2831, Rodríguez \& Foster 705 y Hartshorn \& Meneces 2202.

ObSERVACIONES: Esta especie se reconoce por sus hojas discoloras después del secado, por lo que frecuentemente sus especímenes son erróneamente identificados como S. eichlerii K. Schum., no obstante se diferencian porque esta última especie posee en el envés un indumento fino y abundante, ligeramente de color blancuzco (Fig. 2D). Adicionalmente $S$. tuerckheimii se distingue por sus hojas con margen dentado hacia el ápice, estípulas más o menos persistentes con el desarrollo de las hojas, con vena media, los pecíolos presentan una línea (penacho) de indumento en la superficie adaxial y cápsulas con espinas de hasta $2,5 \mathrm{~cm}$ de longitud.

Material estudiado: BOLIVIA. Beni, Ballivian, Chimanes, 280 m, 12-IX-1978 (fl), Hartshorn \& Meneces 2202 (BOLV). Cochabamaba, Carrasco, Valle de Sacta, $17^{\circ} 05^{\prime} 18^{\prime \prime} \mathrm{S}, 64^{\circ}$ 46'06' 'W, 390 m, 23-X-2007 (fr), Zárate \& Zurita 2767 (LPB, USZ); $17^{\circ} 05^{\prime} 09^{\prime \prime} \mathrm{S}, 64^{\circ} 46^{\prime} 02^{\prime \prime} \mathrm{W}, 390 \mathrm{~m}, 27-\mathrm{IX}-$ 2007 (fr), Zárate \& Vargas 2557 (BOLV); 17 05'16" S, $64^{\circ} 45^{\prime} 59^{\prime \prime} \mathrm{W}, 390$ m, 8-I-200 (fr), Zárate \& Villca 2831 (USZ); Estación experimental Valle de Sacta, Universidad San Simón, $17^{\circ} 05^{\prime} \mathrm{S}, 64^{\circ} 40^{\prime} \mathrm{W}, 288$ m, 14-19-X-1991

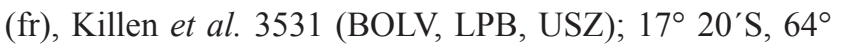
$50^{\prime} \mathrm{W}, 30-\mathrm{X}-1991$ (fr), Galarza et al. 40 (BOLV); $17^{\circ} 20^{\prime} \mathrm{S}$, $64^{\circ} 50^{\prime} \mathrm{W}, 30-\mathrm{X}-1991$, (fr), Galarza 565 (USZ); $17^{\circ} 20^{\prime} \mathrm{S}$, $64^{\circ} 50^{\prime} \mathrm{W}, 335 \mathrm{~m}, 28-\mathrm{X}-1991$ (fr), Villegas \& Cornejo 157 (BOLV). La Paz, Franz Tamayo, Parque Nacional Madidi,

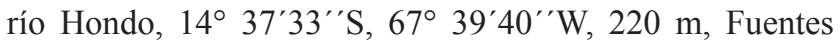
et al. 4336 (LPB). Santa Cruz, Velazco, Parque Nacional Noel Kempff, campamento Los Fierros, $14^{\circ} 33^{\prime} 30^{\prime \prime} \mathrm{S}, 60^{\circ}$ 49'12' W, 155 m, 6-XI-1993 (fl), Quevedo \& Gutiérrez 2481 (LPB); 14 34'56" S, 60 50'01'”, 260 m, 6-XI-1995 (fl, pas. ant.), Rodríguez \& Foster 705 (LPB); campamento la torre, 13 39'20' S, 60 49'08' W, 200 m, 27-XI-1993 (fl), Arroyo et al. 566 (LPB); Parque Nacional Noel Kempff, 14 $\mathrm{km}$ SE de la Estación Flor de Oro, cerca del río Itenez, $13^{\circ}$ $30^{\prime} \mathrm{S}, 61^{\circ} 00^{\prime} \mathrm{W}, 260 \mathrm{~m}, 25-\mathrm{III}-1992$ (st.), Perry \& Cuellar 818 (USZ).

\section{AGRADECIMIENTOS}

Agradecemos al personal del Herbario de la Universidad de Concepción (CONC), al Ministerio de Educación del Gobierno de Chile y al programa de becas MECESUP, por apoyar los estudios de Doctorado en Ciencias Biológicas área Botánica del primer autor, en la Universidad de Concepción y a los curadores de los herbarios BOLV, LPB y USZ, por el préstamo del material vegetal o el envío de imágenes de las colecciones.

\section{BIBLIOGRAFÍA}

Alfaro Castañeda, M.D. 1981. Revisão do gênero Sloanea Linnaeus (Elaeocarpaceae) na Amazônia Brasileira. Tesis de grado. Fundação Universidade do Amazonas. 248 pp.

CRISCI, J. 1994. La especie: realidad y conceptos. En: J. LlorenteBousquets \& I. Luna (eds.), Taxonomía biológica, Vol. 1, pp. 53-64. Universidad Autónoma de México-Fondo de Cultura Económica. México D. F.

D'Arcy, W.G. 1987. Flora of Panama: Checklist and index. Part I. Monographs in Systematic Botany from the Missouri Botanical Garden. 17: Saint Louis, Missouri. 328 pp.

Davis, P.H. \& V.H. Heywood. 1963. Principles of Angiosperm taxonomy. Van Nostrand, New York. 556 pp.

Font Quer, P. 2001. Diccionario de Botánica. Segunda edición. Ediciones Península, S. A., Barcelona. 1244 pp.

JARAmillo, J. 1999. Elaeocarpaceae. En: P.M. Jørgensen \& S. León-Yánez (eds.), Catálogo de las Plantas Vasculares de Ecuador, Vol. 75, pp. 440-441. Monographs in Systematic Botany from the Missouri Botanical Garden.

LIESNER, R. 1993. Elaeocarpaceae. En: L. Brako \& J. Zarucchi (eds.), Catálogo de las Angiospermas y Gimnospermas del Perú, Vol. 45, pp. 413-414. Monographs in Systematic Botany from the Missouri Botanical Garden.

Palacios-Duque, L. 2009. Revisión taxonómica de la sección Brevispicae del género Sloanea L. (Elaeocarpaceae) en Colombia. Tesis de maestría, Universidad Nacional de Colombia, Bogotá, Colombia. 97 pp.

Palacios-Duque, L., C.M. Baeza \& E. Ruíz. 2012. La sección Sloanea del género Sloanea (Elaeocarpaceae) en Bolivia. Revista de la Sociedad Boliviana de Botánica 6(2): 55-61.

Palacios-Duque, L. \& J.L. Fernández-Alonso. 2005. Una nueva e interesante especie de Sloanea (Elaeocarpaceae) del Pacífico colombiano. Revista de la Academia Colombiana de Ciencias Exactas, Físicas y Naturales 29: 179-182.

Palacios-Duque, L. \& J.L. Fernández-Alonso. 2007. Novedades corológicas en Sloanea (Elaeocarpaceae) para Colombia. Revista de la Academia Colombiana de Ciencias Exactas, Físicas y Naturales 31: 29-39.

Palacios-Duque, L. \& J.L. FernándeZ-Alonso. 2012. Dos nuevas especies colombianas de la sección Brevispicae del género Sloanea (Elaeocarpaceae). Anales del Jardín Botánico de Madrid 69(1): 91-95.

SAmpaIO, D. 2009. Revisão das espécies neotropicais extraamazonicas de Sloanea L. (Elaeocarpaceae) na América do Sul. Tese de doutorado, Universidade Estadual de Campinas, Instituto de Biologia, Campinas, São Paulo. 185 pp.

SAmpaIO, D. 2010. Sloanea in Lista de especies da flora do Brasil (online). http://floradobrasil.jbrj.gov.br/2010/FB022649. Consultado: Agosto 11, 2011.

Sampaio, D. \& V.C. SouzA. 2011. Three new species of Sloanea (Elaeocarpaceae). Phytotaxa 16: 45-51.

Smith, C.E. 1954. The new world species of Sloanea (Elaeocarpaceae). Contributions from the Gray Herbarium of Harvard University. 175: 1-114.

Sмiтн, C.E. 1965. Elaeocarpaceae. In: R. E. Woodson, R. W. Scherry \& Collaborators (eds.), Flora of Panama Part VI. Annals of the Missouri Botanical Garden 52(4): 487-495.

Smith, D.A. 1996. Three Previously Undescribed Central 
American Species of Sloanea (Elaeocarpaceae). Novon 6: 120-127.

Sмith, D.A. 2001. Elaeocarpaceae. En: W.D. Stevens, C. UlloaUlloa, A. Pool \& O.M. Montiel (eds.), Flora de Nicaragua, Vol. 85, pp. 816-820. Monographs in Systematic Botany, Missouri Botanical Garden Press, Saint Louis.

Smith, D.A. \& J. Steyermark. 1998. Elaeocarpaceae. In: P.E. Berry, B.K. Holstm \& K. Yatskievych (eds.), Flora of the Venezuelan Guayana, Vol. 4, pp. 712-729. Missouri Botanical Garden Press, Saint Louis.

Steyermark, J.A. 1988. Flora of the Venezuelan Guayana - VI. Annals of the Missouri Botanical Garden 75(4): 1565-
1586.

THIERS, B. 2011. Index Herbariorum: A global directory of public herbaria and associated staff. New York Botanical Garden's Virtual Herbarium. URL: http://sweetgum.nybg. org/ih/. Consultado: Agosto 11, 2011.

Uribe-Meléndez, J. 2008. Monografía de Frullania subgénero Meteoriopsis (Frullaniaceae, Marchantiophyta). Caldasia 30: 49-94.

VÁsquez, R. 2009. Sinopsis del género Sloanea L. (Elaeocarpaceae) en el Perú. Arnaldoa 16(2): 37-63.

Zamora, N., Q. Jiménez \& L.J. Poveda. 2004. Árboles de Costa Rica Volumen III. Ed. INBio. 556 pp.

Recibido: 29.11 .12

Aceptado: 23.05 .13 